Nloman 2021, 39(1)

Revista de Psicologia, Ciències de l'Eduació i de l'Esport

ISSN: 1138-3194

Facultat de Psicologia, Ciències de l'Educació i de l’Esport Blanquerna

Universitat Ramon Llull

\title{
Evolución del uso de las TIC por parte de los adolescentes en los últimos años: luces y sombras
}

\author{
Jesús Plaza de la Hoz \\ Universidad Internacional de La Rioja (UNIR)
}

Recibido: 2020-7-23

Aceptado: 2021-2-8

doi: 10.51698/aloma.2021.39.1.39-47

Evolución del uso de las TIC por parte de los adolescentes en los últimos años: luces y sombras

\begin{abstract}
INTRODUCCIÓN. Estudios recientes muestran que el uso de las tecnologías de la información y la comunicación (TIC) se generaliza entre los adolescentes y que persisten los inconvenientes derivados; de ahí, el interés por la evolución de esta relación jóvenes-tecnologías. MÉTODO. Este artículo compara resultados obtenidos en 2013 con un cuestionario cumplimentado por una muestra representativa (1.032 estudiantes de ESO) con otra más reducida (235) de similar alumnado tomada en 2019. El objetivo es observar los cambios sobre el uso de Internet, su influjo en el rendimiento académico y la vulnerabilidad de los jóvenes frente a las TIC. Para la comparativa se usa el programa de tratamiento de datos SPSS, aunque con un alcance exploratorio. RESULTADOS. Se observa una disminución tanto de efectos positivos como negativos; se constata también un uso prudente de la red, pero un mayor porcentaje de los que sienten amenazas. Además, los afectados sufren en solitario sus problemas en mayor grado. DISCUSIÓN. Se confirma la normalización del uso de TIC advertido en estudios recientes, junto con efectos negativos diversos. Ante esta doble tendencia, conviene seguir empoderando a los adolescentes en usar responsablemente las $T I C$, de modo que se normalice también la petición de ayuda, especialmente por parte de los afectados más negativamente.
\end{abstract}

Palabras clave: educación; tecnologías de la información y comunicación; adolescencia; cambio de actitud; relaciones entre pares.

The evolution of ICT use by adolescents in recent years: lights and shadows

\begin{abstract}
INTRODUCTION. Recent studies show a generalized use of new technologies (ICT) among adolescents, and the persistence of related disadvantages. Therefore, the evolution of this relationship between young people and technology is worthy of scholarly attention. METHOD. This article compares results obtained in 2013 via a survey completed by a representative sample (1032 participants) of students of compulsory education with the results of a survey with a smaller smaple (235) of similar students completed in 2019. The aim is to observe changes in the use of Internet, its impact on academic performance, and perceived vulnerability to ICT risks. For this comparison, the Statistical Package for the Social Sciences (SPSS) is used, albeit in an exploratory manner. RESULTS. The study showed decreases in both the positive and negative effects of Internet use perceived by the students. It also shows an overall more prudent use of the Internet in the more recent survey, although more students in this survey reported being subjected to online threats. The victims of these threats were also more likely to undergo these problems alone. DISCUSSION. The study confirms the normalization of Internet use shown in previous research, along with a number of negative effects. In light of this dual trend, it seems to be desirable to continue to empower adolescents to make a responsible use of ICT while encouraging those who are affected in a negative way to ask for help.
\end{abstract}

Keywords: education; information and communication technologies; adolescence; attitude change; peer relationship.

Correspondencia

Jesús Plaza de la Hoz

ORCID: 0000-0002-6966-4214

Universidad Internacional de la Rioja (UNIR)

jesus.plaza@unir.net 


\section{Introducción}

Es común comentar que los adolescentes españoles hacen un uso generalizado de las tecnologías de la información y la comunicación (TIC). Dicho uso incluye navegar por el complejo mundo de Internet, utilizar telefonía móvil de última generación y tener perfiles en diversos canales de comunicación y redes sociales virtuales disponibles. Las estadísticas oficiales al respecto (INE, 2019) señalan que más del 90\% de los hogares españoles disponen de acceso a Internet; además, un tanto por ciento similar de población de 14 años dispone de móvil (en realidad, desde los 12 años ya lo utilizan un $64 \%$ de niños y niñas en España). Una situación similar a lo que muestra el contexto internacional y que desde hace algunos años se constata desde la etapa preescolar (Crescenzi et al., 2014; Lepicnik \& Samec, 2013; Plowman et al., 2012).

Las funcionalidades inmediatas de las TIC, informar y comunicar, se despliegan en la gestión de las interacciones. A través de las redes sociales, se canalizan nuevos modos de entretenerse y divertirse; se multiplican los objetivos de la búsqueda de información -educativo, lúdico, laboral, de consumo, cultural, etc.-; el usuario es ahora productor de contenidos -foros, blogs, webs, canales de producción de vídeo, etc.-- Se puede hablar, en este sentido, de una continua expansión de los usos (Megías, 2019; Megías \& Rodríguez, 2018) que hace que en la vivencia de los jóvenes primen más las ventajas que los inconvenientes, en línea con una globalización asumida de la progresiva e imparable digitalización de la sociedad.

Así pues, el impacto que tienen las TIC en los adolescentes es innegable y está siendo objeto de interés científico constante desde hace una década también en el ámbito español (Colás et al., 2013; Colás et al., 2018; Espinar y Fernández, 2009; Fernández-Montalvo et al., 2015). A este respecto, se han documentado muchos aspectos positivos diferenciales en cuanto a la mejora del aprendizaje y el rendimiento académicos (García-Martín \& Cantón-Mayo, 2019; Muñoz-Miralles et al., 2014; Valencia-Peris et al., 2016). Así, la búsqueda de información, la confección de tareas y trabajos, los tutoriales que amplían la explicación recibida en el aula, etc. pueden ser de gran utilidad académica; además, las TIC favorecen la motivación, un aprendizaje más autónomo y un estilo de docencia más innovador y participativo; cuando su uso es moderado, pueden facilitar y conservar las relaciones sociales y el contacto con familiares y amigos.

Y a la vez, se reconocen los riesgos que lleva aparejada una utilización no adecuada de estos medios (Díaz-Vicario et al, 2019; Méndez-Gago \& GonzálezRobledo, 2018), como son el abuso, la dependencia y las conductas problemáticas que afectan a los entornos familiar, sociorrelacional y académico. En la familia, las TIC pueden distorsionar las relaciones y actividades comunes cuando se usan en exceso o inoportunamente; el tiempo invertido en el mundo virtual reduce el tiempo disponible para actividades culturales, de es- parcimiento o deportivas con los iguales; dan lugar a malentendidos y pueden utilizarse fácilmente con intención de hacer daño; finalmente, para algunos supone perder el tiempo y bajar su rendimiento escolar.

El público adolescente es especialmente objeto de interés cuando se trata de TIC, por estar en una etapa evolutiva de formación de la personalidad y forja de la identidad, en la que priman las relaciones con sus iguales, el atractivo de las novedades, el afán de experimentar y de arriesgarse.

Dada la evolución de las tecnologías como catalizadores de rápidos cambios sociales (Grande et al., 2016), es pertinente poner el foco de atención en el desarrollo de los hábitos de uso que los adolescentes manifiestan cuando se les pregunta específicamente. En la medida en que forman parte de la vida cotidiana, familiar, académica y de ocio, conviene seguir recogiendo información de cómo influyen las TIC en una etapa evolutiva de gran importancia. En efecto, la adolescencia supone tantas veces la primera forja de la identidad personal, del proyecto profesional futuro y de un contexto familiar y de amistades más amplio; coincide además con etapas de transición educativa (Bernal \& König, 2017), como es el comienzo de la Educación Segundaria Obligatoria en España. Que todas estas importantes relaciones estén mediadas por las TIC implica una gran responsabilidad por parte de padres, profesores e investigadores sociales; conviene analizar su impacto, prevenir sus inconvenientes y concienciar a los jóvenes de hoy sobre el uso adecuado y empoderador de la tecnología; así serán dueños de su futuro y no meros consumidores en la estructura del mercado, o incluso víctimas de estas potentes herramientas.

Por todo ello, sigue siendo pertinente llevar a cabo estudios comparativos en el tiempo, para observar la evolución del uso que dicen hacer los jóvenes de las TIC, tanto en los entornos de ocio como escolares; de ese modo se podrá comprobar el impacto positivo que tienen en sus vidas y los efectos no deseados de su utilización; finalmente, arrojará luz sobre el modo cómo se enfrentan los adolescentes a los riesgos aparejados. Con ello, será viable hacer propuestas realistas para reorientar su influencia desde las instancias familiar y docente.

\section{Objetivos del presente estudio}

La finalidad de este artículo es observar si han cambiado y en qué medida las motivaciones que llevan a los adolescentes a usar las TIC, las percepciones de posibles amenazas a las que se exponen, así como su manera de responder ante estas.

Concretamente, los objetivos específicos se especifican como sigue:

- Observar la variación del impacto que el uso de las TIC ha supuesto para los adolescentes españoles de 12 a 17 años entre 2013 y 2019, en opinión de los propios jóvenes.

- Advertir si ha mejorado el grado de responsabilidad/vulnerabilidad en la red de redes percibida por los adolescentes en este período. 
- Comprobar en qué medida están hoy más expuestos a amenazas reales que hace cinco años, según su apreciación.

- Analizar si en estos años se ha modificado el modo de reaccionar de los adolescentes españoles ante esas amenazas ocasionadas por el uso intensivo de las TIC.

\section{Método}

Se trata de un estudio comparativo de datos cuantitativos que fueron recogidos tras la aplicación de un cuestionario acerca del uso habitual que hacían los adolescentes de las TIC, de los retos éticos a los que se enfrentaban con estas, de las amenazas que percibían y cómo se enfrentaban. Participó un grupo de 235 estudiantes de entre 12 y 17 años (229 válidos, 142 chicos y 87 chicas) que cursaban de $1 .^{\circ}$ a $4 .^{\circ}$ de ESO en el momento de cumplimentarlo (finales de 2018 y principios de 2019).

Los datos de la muestra (Tabla 1) reflejan la diversidad intencional de titularidad de los centros escolares (un instituto público de Teruel y un colegio concertado de Logroño) y de sistemas pedagógicos (uno con uso de TIC en el aula como instrumento de apoyo; otro con el sistema 1:1 de un iPad por alumno). Asimismo, se tomaron referencias de su perfil sociodemográfico, modos de uso de Internet y apreciación de conductas de riesgo en la Red.

Se contó como referencia con los resultados de la misma encuesta llevada a cabo en 2013, con una muestra representativa de 1.032 estudiantes españoles de las mismas edades y similares características (Plaza-de-laHoz, 2016; 2018), con la que se pretendían contrastar algunos de los ítems para observar de modo exploratorio su evolución de los comportamientos en el tiempo transcurrido.

\section{Participantes}

Se trató de un estudio no estadísticamente representativo, con una muestra reducida, que respondía a las posibilidades reales de acceso directo por parte del investigador. Lo formaron 235 estudiantes de $1 .^{\circ}$ a $4 .^{\circ}$ de ESO de dos centros educativos (uno público y otro concertado), de dos comunidades autónomas (Aragón y La Rioja), de variado espectro social (clase mediamedia alta); los participantes rellenaron el cuestionario voluntariamente, aprovechando los horarios de algunas clases y contando con el visto bueno previo de la dirección de los centros. En este sentido, se observó una cierta sobrerrepresentación de alumnos de $4 .^{\circ}$ de ESO (29.8\%) y de varones $(60.4 \%)$. En cuanto a la calidad de su rendimiento académico, el 50\% de los alumnos encuestados tenían una media de entre 5 y 7 , y tanto los que obtuvieron menos de un 5, como los que obtuvieron más de un 8 fueron el 15\%. En cuanto al espectro socioeconómico de la muestra, no recogido en los datos de la tabla, el instituto público turolense, que era uno de los cuatro existentes en la capital, recogía población de variada extracción social, incluso alum-
Tabla 1. Frecuencias de datos básicos sociodemográficos.

\begin{tabular}{|c|c|c|c|c|c|}
\hline & & Frecuencia & Porcentaje & $\begin{array}{l}\text { Porcentaje } \\
\text { válido }\end{array}$ & $\begin{array}{l}\text { Porcentaje } \\
\text { acumulado }\end{array}$ \\
\hline \multicolumn{6}{|l|}{ Sexo } \\
\hline \multirow[t]{4}{*}{ Válido } & Hombre & 142 & 60.4 & 62.0 & 62.0 \\
\hline & Mujer & 87 & 37.0 & 38.0 & 100.0 \\
\hline & Total & 229 & 97.4 & 100.0 & \\
\hline & Perdidos & 6 & 2.6 & & \\
\hline Total & & 235 & 100.0 & & \\
\hline \multicolumn{6}{|c|}{ Titularidad del centro } \\
\hline \multirow[t]{4}{*}{ Válido } & Público & 171 & 72.8 & 73.1 & 73.1 \\
\hline & Concertado & 63 & 26.8 & 26.9 & 100.0 \\
\hline & Total & 234 & 99.6 & 100.0 & \\
\hline & Perdidos & 1 & 0.4 & & \\
\hline Total & & 235 & 100.0 & & \\
\hline \multicolumn{6}{|l|}{ Curso } \\
\hline \multirow[t]{6}{*}{ Válido } & 1. ${ }^{\circ} \mathrm{ESO}$ & 56 & 23.8 & 23.9 & 23.9 \\
\hline & $2 .^{\circ} \mathrm{ESO}$ & 52 & 22.1 & 22.2 & 46.2 \\
\hline & $3 .^{\circ} \mathrm{ESO}$ & 56 & 23.8 & 23.9 & 70.1 \\
\hline & $4 .^{\circ} \mathrm{ESO}$ & 70 & 29.8 & 29.9 & 100.0 \\
\hline & Total & 234 & 99.6 & 100.0 & \\
\hline & Perdidos & 1 & 0.4 & & \\
\hline Total & & 235 & 100.0 & & \\
\hline \multicolumn{6}{|c|}{ Número de hermanos } \\
\hline \multirow[t]{7}{*}{ Válido } & Ninguno & 24 & 10.2 & 10.7 & 10.7 \\
\hline & Uno & 131 & 55.7 & 58.5 & 69.2 \\
\hline & Dos & 51 & 21.7 & 22.8 & 92.0 \\
\hline & Tres & 11 & 4.7 & 4.9 & 96.9 \\
\hline & Más de tres & 7 & 3.0 & 3.1 & 100.0 \\
\hline & Total & 224 & 95.3 & 100.0 & \\
\hline & Perdidos & 11 & 4.7 & & \\
\hline Total & & 235 & 100.0 & & \\
\hline \multicolumn{6}{|c|}{ Nota media } \\
\hline \multirow[t]{9}{*}{ Válido } & Menos de 5 & 36 & 15.3 & 15.5 & 15.5 \\
\hline & Entre 5 y 6 & 70 & 29.8 & 30.0 & 45.5 \\
\hline & Entre 6 y 7 & 45 & 19.1 & 19.3 & 64.8 \\
\hline & Entre 7 y 8 & 41 & 17.4 & 17.6 & 82.4 \\
\hline & Entre 8 y 9 & 24 & 10.2 & 10.3 & 92.7 \\
\hline & Entre 9 y 10 & 16 & 6.8 & 6.9 & 99.6 \\
\hline & 22 & 1 & 0.4 & 0.4 & 100.0 \\
\hline & Total & 233 & 99.1 & 100.0 & \\
\hline & Perdidos & 2 & 0.9 & & \\
\hline Total & & 235 & 100.0 & & \\
\hline
\end{tabular}

nado rural de poblaciones cercanas. En cuanto al centro concertado, su alumnado respondía a un perfil familiar de clase media-alta. Por tanto, aun tratándose de una muestra no muy amplia respondía a un casillero tipológico medio.

\section{Instrumento y análisis de la información}

El cuestionario para la recogida de los datos («Internet, redes sociales y educación») fue diseñado de modo específico para la investigación llevada a cabo en 2013, en la que intervinieron ocho profesores de cuatro universidades distintas, tanto en su confección como en su aplicación y explotación de los datos. Se replicó con mínimas variaciones de algún término (la inclusión de algunas redes sociales que eran más actuales que entonces, como Instagram o Snapchat). Constó de 51 ítems a través de los cuales se preguntó sobre el uso, el tipo y la intensidad de manejo de las TIC en general y 


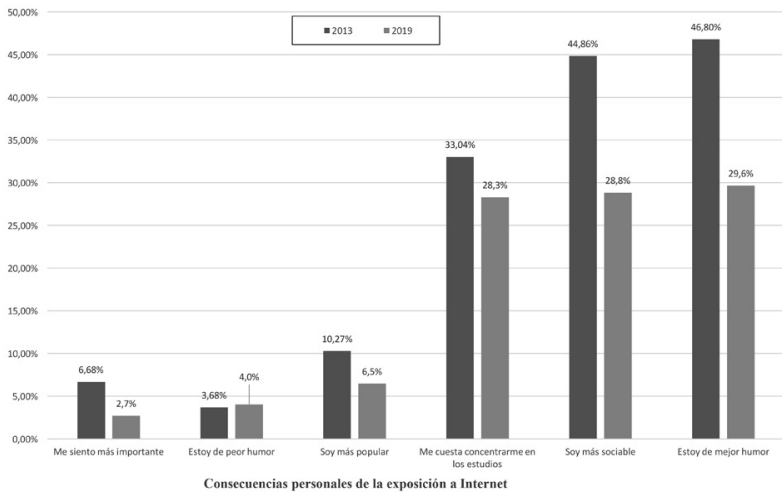

Figura 1. Logros obtenidos gracias a Internet Fuente: elaboración propia a partir del ítem P38 del cuestionario «Internet, redes sociales y educación» (2013-2019).

de redes sociales en particular. Se escogieron 8 ítems (ver Anexo 1) relacionados con los objetivos de este estudio: motivaciones, medidas de seguridad, conciencia de riesgos, exposición a amenazas y modo de reaccionar ante estas.

Los datos se recogieron entre 2018 y 2019, tras ser administrados personalmente por el autor de este artículo a un grupo de 235 alumnos de los centros mencionados. Al tratarse de un estudio descriptivo exploratorio, no se pretendía un alcance estadístico de los resultados, aunque sí se procuró reflejar en la muestra variedad de situaciones en cuanto a sexo, edades, titularidad del centro, composición familiar y rendimiento del alumnado.

Los resultados obtenidos se distribuyeron en tablas con el programa informático SPSS Statistics 25, de un modo similar a como se hizo con los resultados del estudio de 2013, de modo que pudieran establecerse comparaciones entre los porcentajes de respuestas.

\section{Resultados}

\section{Se modera el impacto del uso de las TIC apreciado} por los adolescentes

En la Figura 1 se muestra la variación en cuanto a cómo apreciaban los encuestados que les influyeran las tecnologías. Puede advertirse que se moderaban todos los índices, tanto positivos (me siento importante, más sociable, de mejor humor, más popular), como negativos (me cuesta concentrarme, estoy de peor humor). Tratándose de preguntas con respuesta múltiple, se notó un descenso en todas las opciones positivas en mayor grado que en las negativas; incluso creció algo la opción «estoy de peor humor».

\section{Cambios en la responsabilidad/vulnerabilidad percibida en la red}

En primer lugar, conviene indicar que, en lo esencial, los adolescentes se comportaron con las TIC de un modo similar, independientemente de su condición de vulnerabilidad personal (Melendro et al., 2016). Sin embargo, a tenor de los datos recogidos en la Figura 2 sobre comportamiento responsable en Internet, pode-

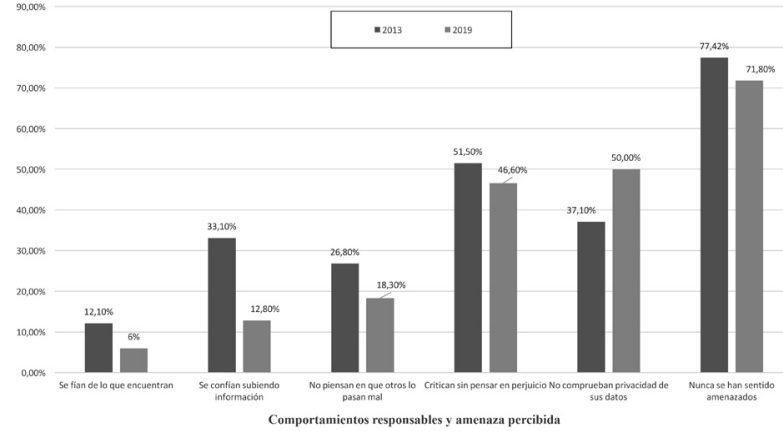

Figura 2. Vulnerabilidad percibida en Internet

Fuente: elaboración propia a partir de datos de los ítems P6, P12, P15, P20, P25 y P50 del cuestionario «Internet, redes sociales y educación» (2013-2019).

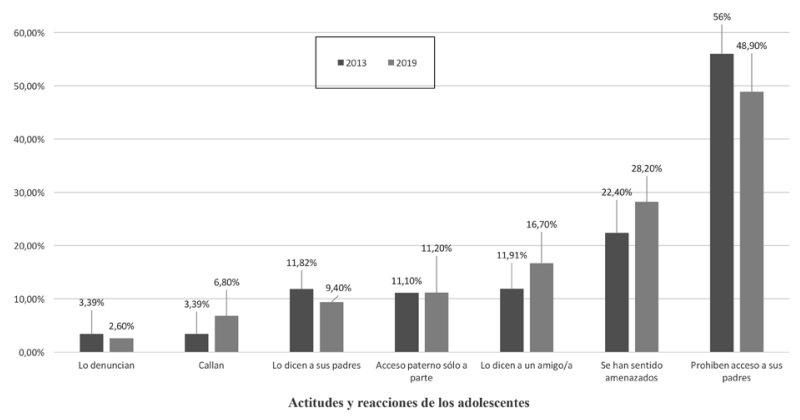

Figura 3. Exposición a riesgos en la red

Fuente: elaboración propia a partir de datos de los ítems P13 y P20 del cuestionario Fuente: elaboración propia a partir de datos de los

mos observar un cambio en esa vulnerabilidad general de los adolescentes. Si se pone el foco de atención en los aspectos positivos, disminuyó a la mitad el número de los estudiantes que se fiaban de los contenidos de Internet y descendieron en 20 puntos los que en 2013 se confiaban subiendo información. Por otra parte, aun disminuyendo algo, todavía un $46.6 \%$ criticaba sin pensar en el daño que podía causar a otros usuarios, así como ascendió, desde un 37.1\% hasta la mitad de la muestra, los que no comprobaban la privacidad de sus datos; por último, se incrementó hasta un 28.2\% los que se habían sentido amenazados alguna vez cuando cinco años atrás era de un $22.4 \%$.

Valoración diversa del comportamiento adoptado respecto a los riesgos en Internet

En la Figura 3 vemos en qué medida variaron los comportamientos de los adolescentes que se enfrentaban a riesgos derivados de las TIC. Por una parte, se incrementó el acceso de los padres a las redes y perfiles de los hijos, aunque fuera solo a parte de esa información. Por otra, creció el número de los que se sintieron amenazados, los que confiaban más en los amigos y amigas que en los padres y, sobre todo, se duplicó el número de los que callaban y no lo contaban a nadie, de un $3.39 \%$ a un $6.80 \%$.

Al desagrupar los datos de 2013 y de 2019 atendiendo a los diferentes cursos de los participantes en el cuestionario (Tabla 2), advertimos también algunas variaciones de interés.

En primer lugar, en todos los cursos aumentó la connivencia con los amigos en caso de amenaza; fue en $3 .^{\circ}$ de ESO donde se produjo un mayor incremento 
Tabla 2. ¿Qué hiciste al sentirte amenazado a través de Internet?

\begin{tabular}{|c|c|c|c|c|c|c|c|c|c|c|c|}
\hline & & \multicolumn{8}{|c|}{ Curso } & \multirow{2}{*}{\multicolumn{2}{|c|}{ Total }} \\
\hline & & \multicolumn{2}{|c|}{$1 .^{\circ} \mathrm{ESO}$} & \multicolumn{2}{|c|}{$2 .^{\circ} \mathrm{ESO}$} & \multicolumn{2}{|c|}{$3 .^{\circ} \mathrm{ESO}$} & \multicolumn{2}{|c|}{$4 .^{\circ} \mathrm{ESO}$} & & \\
\hline & & 2013 & 2019 & 2013 & 2019 & 2013 & 2019 & 2013 & 2019 & 2013 & 2019 \\
\hline \multirow{3}{*}{$\begin{array}{l}\text { Acudí a alguna } \\
\text { amistad }\end{array}$} & Recuento & & 7 & & 8 & & 12 & & 12 & & 39 \\
\hline & $\%$ dentro de curso & $8.30 \%$ & $12.5 \%$ & $11.6 \%$ & $15.4 \%$ & $12.4 \%$ & $21.4 \%$ & $13.1 \%$ & $17.1 \%$ & & \\
\hline & $\%$ del total & & $3.0 \%$ & & $3.4 \%$ & & $5.1 \%$ & & $5.1 \%$ & $12.0 \%$ & $16.7 \%$ \\
\hline \multirow{3}{*}{$\begin{array}{l}\text { Acudí a mis } \\
\text { padres }\end{array}$} & Recuento & & 6 & & 3 & & 7 & & 6 & & 22 \\
\hline & \% dentro de curso & $13.40 \%$ & $10.7 \%$ & $12.8 \%$ & $5.8 \%$ & $12.7 \%$ & $12.5 \%$ & $9.8 \%$ & $8.6 \%$ & & \\
\hline & $\%$ del total & & $2.6 \%$ & & $1.3 \%$ & & $3.0 \%$ & & $2.6 \%$ & $11.8 \%$ & $9.4 \%$ \\
\hline \multirow[t]{3}{*}{ Acudí a un adulto } & Recuento & & 2 & & 0 & & 1 & & 3 & & 6 \\
\hline & $\%$ dentro de curso & $3.80 \%$ & $3.6 \%$ & $5.0 \%$ & $0.0 \%$ & $1.6 \%$ & $1.8 \%$ & $3.6 \%$ & $4.3 \%$ & & \\
\hline & $\%$ del total & & $0.9 \%$ & & $0.0 \%$ & & $0.4 \%$ & & $1.3 \%$ & $3.3 \%$ & $2.6 \%$ \\
\hline \multirow{3}{*}{$\begin{array}{l}\text { Lo denuncié al } \\
\text { colegio }\end{array}$} & Recuento & & 1 & & 0 & & 2 & & 3 & & 6 \\
\hline & $\%$ dentro de curso & $2.50 \%$ & $1.8 \%$ & $2.7 \%$ & $0.0 \%$ & $3.6 \%$ & $3.6 \%$ & $4.1 \%$ & $4.3 \%$ & & \\
\hline & $\%$ del total & & $0.4 \%$ & & $0.0 \%$ & & $0.9 \%$ & & $1.3 \%$ & $3.4 \%$ & $2.6 \%$ \\
\hline \multirow{3}{*}{$\begin{array}{l}\text { No lo comenté } \\
\text { con nadie }\end{array}$} & Recuento & & 4 & & 6 & & 3 & & 3 & & 16 \\
\hline & $\%$ dentro de curso & $3.50 \%$ & $7.1 \%$ & $2.9 \%$ & $11.5 \%$ & $3.2 \%$ & $5.4 \%$ & $3.8 \%$ & $4.3 \%$ & & \\
\hline & $\%$ del total & & $1.7 \%$ & & $2.6 \%$ & & $1.3 \%$ & & $1.3 \%$ & $3.4 \%$ & $6.8 \%$ \\
\hline \multirow{3}{*}{$\begin{array}{l}\text { Nunca me he } \\
\text { sentido } \\
\text { amenazado }\end{array}$} & Recuento & & 42 & & 41 & & 37 & & 48 & & 168 \\
\hline & $\%$ dentro de curso & $78.50 \%$ & $75.0 \%$ & $78.1 \%$ & $78.8 \%$ & $76.4 \%$ & $66.1 \%$ & $77.9 \%$ & $68,6 \%$ & & \\
\hline & $\%$ del total & & $17.9 \%$ & & $17.5 \%$ & & $15.8 \%$ & & $20.5 \%$ & $77.4 \%$ & $71.8 \%$ \\
\hline \multirow[t]{2}{*}{ Total } & Recuento & & 56 & & 52 & & 56 & & 70 & & 234 \\
\hline & $\%$ del total & & $23.9 \%$ & & $22.2 \%$ & & $23.9 \%$ & & $29.9 \%$ & & $100.0 \%$ \\
\hline
\end{tabular}

Fuente: elaboración propia a partir del ítem P20 del cuestionario «Internet, redes sociales y educación» (2013-2019).

(de 8 puntos). Por otra parte, la disminución de 2 puntos porcentuales en pedir ayuda y consejo a los padres se elevó hasta los 7 puntos en el caso del alumnado de $2 .^{\circ}$ de ESO. A la hora de sufrir en solitario las amenazas -no lo comentaban con nadie-, fueron los pequeños (1. ${ }^{\circ}$ y $2 .^{\circ}$ de ESO) los que más aumentaron su vulnerabilidad respecto a 2013, en 4 y 8 puntos respectivamente. Finalmente, fueron los mayores, de $3 .^{\circ} \mathrm{y} 4 .^{\circ}$ de ESO, los que más reconocieron haberse sentido amenazados en alguna ocasión (casi 10 puntos por encima respecto a los datos de 2013).

Empeoran las reacciones del grupo de los amenazados alguna vez en las redes

Si se centra la atención en el grupo de alumnado que reconoció haberse sentido amenazado en alguna ocasión (Figura 4), se puede observar que disminuyeron en casi 20 puntos los que acudieron a sus padres, mientras que solo aumentaron en 5.4 puntos los que confiaban en un amigo o una amiga. En definitiva, este

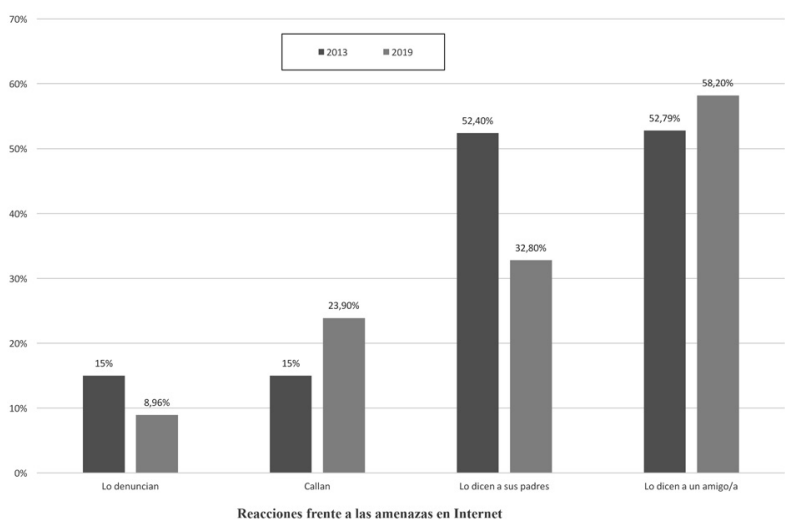

Figura 4. Actitud ante las amenazas Fuente: elaboración propia a partir del ítem P20 del cuestionario «Internet, redes sociales y educación» (2013-2019). grupo de alumnado denunciaba menos y callaba más que cinco años atrás.

\section{Discusión y conclusiones}

La Unión Europea (European Union, 2015), en su informe de hace unos años, advertía acerca del incremento tanto del tiempo que los jóvenes dedican al mundo digital, como de los riesgos provocados por las TIC entre los más vulnerables. Los resultados del estudio comparativo que aquí se ofrecen hablan de una cierta moderación en la percepción adolescente de este influjo. Al darse ese descenso tanto en los aspectos positivos como en los negativos, los jóvenes pueden estar normalizando su relación con las tecnologías, en un momento en que su uso alcanza la saturación, se adelanta cada vez más en su inicio y se intensifica en la etapa adolescente que se contempla aquí. En este sentido, parece que las TIC dejan de ser herramientas novedosas y pasan a formar parte del contexto de bienes de consumo comunes y aun de uso educativo con enormes posibilidades ( $\mathrm{Hu}, 2017$; OECD, 2015).

Junto con ello, sigue siendo importante que un $28 \%$ de los encuestados considere las TIC como un elemento de distracción para el estudio, lo que confirma los resultados recogidos en investigaciones anteriores (Beland \& Murphy, 2015; Haddon \& Vincent, 2014). Porque, aunque los datos de 2019 son inferiores en 5 puntos respecto a la percepción de hace cinco años (a un 33.04\% le costaba concentrase por causa de las TIC), es un descenso mucho menor que el manifestado respecto a sus connotaciones sociales positivas. Estas últimas quizá se vieron alentadas estos años por una publicidad que las elevaba a panacea de éxito adolescente a través de la propia imagen compartida. 
En cuanto a los comportamientos relacionados con el aspecto ético, los estudiantes encuestados parecen enfrentarse con más precaución a los contenidos de la red y ser algo más conscientes de sus posibles efectos negativos. Es posible que por esa razón - paradójicamente- se eleven hasta la mitad los que descuidan su propia privacidad; algo que podría estar relacionado con la baja percepción del riesgo respecto al uso de TIC que indican autores como Alfaro et al. (2015). Como consecuencia, aumentan en más de 5 puntos los alumnos y las alumnas que han percibido amenazas; una ambigüedad que ya señalaban Rial et al. (2014), Ballesteros y Megías (2015) y, más recientemente, Díaz-Vicario et al. (2019).

En cuanto al porcentaje total que ha sido amenazado alguna vez (28.2\%), el incremento que se observa en estos cinco años se equipara al 29\% advertido por Buelga et al. (2010), lo cual no puede ser interpretado como un retroceso, pero sí como un estancamiento que justifica manifestar cierta preocupación, puesto que apenas disminuye al cabo de estos años.

Quizá la mencionada progresiva normalización del uso de TIC puede contribuir a esa mayor exposición a los riesgos que muestran los datos, si bien los estudios al respecto varían algo en el volumen de su impacto. Psicológicamente, un uso problemático o no adaptativo, como el señalado por Rial et al. (2015), afectaba a un $26.6 \%$ de adolescentes; un uso problemático del móvil obtiene una puntuación alta-severa similar, en un $26.3 \%$ de adolescentes mayores, según un estudio reciente de Ávila et al. (2019); por su parte, Besolí et al. (2018) reducen a un 15\% los adolescentes en los que se advierte el riesgo de un uso problemático. Sin embargo, desde la perspectiva sociológica de este estudio, el uso consciente no adecuado de Internet alcanza un porcentaje bastante mayor, pues el $46.60 \%$ de la muestra critica sin pensar en el perjuicio que causa, si bien parece reducirse algo en la comparativa respecto a 2013 (cuando un 51.50\% señalaba esa opción).

En este sentido, estos mismos datos aportados por el estudio no están lejos de los porcentajes de conductas de riesgo que advertían Gairín y Mercader (2018), en cuanto a que el $31 \%$ de los adolescentes de entre 15 y 17 años reconoce haber molestado a otros durante el último año y un 41.7\% haberse burlado de sus compañeros. Estos autores, sin embargo, no aprecian que se trate de comportamientos aplicables a todos los adolescentes, ni muestran la recurrencia que sugirieron Chóliz y Villanueva (2011); en esta línea, tampoco este cuestionario permite hablar de indicios de adicción en el sentido en que viene definida por la DMS-5 (American Psychiatric Association, 2014). Y, sin embargo, la elevada presencia de estas conductas en este grupo de edad y su persistencia a lo largo del tiempo, permite concluir a partir del presente análisis (coincidente en parte con el de Méndez-Gago y González Robledo, de 2018, que cifra en un 31.5\% los adolescentes que dan señales de un uso inadecuado de Internet; o el de Arnaiz et al., 2016, que apunta al mismo porcentaje en situación de riesgo) que se hace urgente una educación más incisiva en el uso con sentido -tanto social como éticode la red de redes.

Por otra parte, atendiendo a las relaciones del alumnado amenazado a través de las TIC con su entorno de apoyo, si bien parece crecer el control de los padres, disminuye algo su papel de confidentes y, sobre todo, son más los adolescentes que se enfrentan solos ante los problemas. Esta circunstancia reafirma la brecha que puede darse entre las percepciones de actividades de riesgo con las TIC de los padres y de los hijos, como Berrios et al. (2015) muestran en su estudio exploratorio; algo que también ponen de manifiesto Besolí et al. (2018) en su investigación mixta con padres e hijos.

Observando las edades en las que se dan las mayores variaciones (que se indican en la Tabla 2), la confianza en los amigos que muestran los alumnos de $3 .^{\circ}$ de ESO se ha incrementado quizá debido en parte a la sobrerrepresentación de los chicos en la muestra de 2019; téngase en cuenta que las alumnas suelen entrar en la adolescencia en cursos previos, con el mayor peso que pueden tener entonces las relaciones entre iguales.

Sin embargo, si unimos el descenso en el porcentaje de alumnos de $1 .^{\circ}$ y $2 .^{\circ}$ de ESO que recurren a sus padres en caso de amenaza (de 3 a 7 puntos), con el hecho de que son ellos también los que más han aumentado en elegir la opción de no comentarlo con nadie (de 4 a 8 puntos), se confirma lo que Gairín y Mercader (2018) concluían en su reciente estudio, a saber, que es mayor la percepción de soledad ante el uso de las TIC entre el alumnado de 12 a 14 años, que entre aquellos de 15 a 17; también Fernández-Montalvo et al. (2015) mostraban un perfil diferencial de conductas de riesgo en Internet desde la preadolescencia, especialmente entre los chicos. Por último, tanto Arnaiz et al. (2016) como Giménez (2015) y RodríguezÁlvarez et al. (2018), advierten que los problemas con la red y el ciberbullying aparecen ya en los últimos cursos de Primaria.

En cambio, en términos globales, en la muestra de 2019 son los mayores (de $3 .^{\circ}$ y $4 .^{\circ}$ de ESO) los que más reconocen haberse sentido amenazados alguna vez (casi 10 puntos más que en 2013). La explicación puede deberse a múltiples factores, quizá también al hecho de que este cuestionario lo hayan cumplimentado significativamente más varones, cuya mayor tendencia a las conductas de riesgo ha sido mostrada por diversos estudios (Alfaro et al., 2015; Gómez et al., 2018). De igual modo, los adolescentes mayores tienen, en este sentido, más experiencia y posibilidades de haberse encontrado en situaciones comprometidas.

Finalmente, cuando se centra la atención en aquellos estudiantes que reconocen haber sufrido amenazas, se observa que no solo son más en porcentaje, sino que están más expuestos a las consecuencias negativas derivadas, puesto que, si bien recurren más a los amigos que hace cinco años, no lo hacen en la misma medida en que dejan fuera a sus padres; $y$, sobre todo, aumentan los que sufren en solitario situaciones negativas. Esta percepción peyorativa de la ayuda que los padres puedan prestar la situaban Ramos-Soler et al. (2018) en 
el umbral de los estudiantes de 11 años, en el que disminuye la mediación de los padres al tiempo que aumenta la frecuencia e intensidad de uso de las TIC por parte de los menores; en este sentido, sería coincidente con los resultados de este estudio.

En definitiva, de los datos recopilados y de su análisis comparativo exploratorio se puede sugerir que la progresiva normalización de las TIC entre los adolescentes puede estar contribuyendo a moderar la imagen excesivamente idealizada de la que hasta ahora habían gozado en este grupo de edad. Al mismo tiempo, las distintas campañas de concienciación sobre sus riesgos pueden haber facilitado unas actitudes más conscientes desde el punto de vista ético.

Normalización y consciencia de amenazas se combinan paradójicamente con un menor cuidado de la privacidad; es típico del adolescente pensar que a él o a ella no le va a pasar. Quizá por eso aumenta en más de 6 puntos el alumnado que percibió amenazas.

Aunque lo verdaderamente relevante es que más de un $23 \%$ de los amenazados opta por no contárselo a nadie. Y a pesar de que queda por dilucidar la mayor o menor gravedad de esas amenazas, se refuerza la urgencia de seguir concienciando a los jóvenes de la necesidad de que pidan ayuda externa, para visibilizar los comportamientos negativos y prevenir y auxiliar a las víctimas potenciales y actuales.

\section{Limitaciones y prospectiva}

La primera y evidente limitación de este estudio es el tamaño de la muestra que hubiera requerido mayor volumen para alcanzar la representatividad estadística. Sin embargo, el hecho de que se traten las mismas cuestiones, con participantes de las mismas edades y de un perfil sociodemográfico plural sigue siendo útil de modo descriptivo exploratorio. La sobrerrepresentación de los varones, en este caso, aun constituyendo un claro sesgo, se ha tenido en cuenta a la hora de interpretar algunos resultados; sin embargo, debiera ser corregido en posteriores estudios comparativos longitudinales. De igual modo, toda la información recogida procede de los adolescentes, por lo que convendría que se completase con la opinión de padres y profesores, sobre todo en orden a promover tanto unos usos responsables de la red, como la eficacia de la ayuda que los adultos puedan prestar a los menores.

En cuanto a la prospectiva, a la luz de los trabajos tenidos en cuenta en la discusión y la variación misma que este estudio advierte en estos últimos años, se recomienda ahondar en los factores que pueden estar influyendo en la persistencia de conductas de riesgo dentro del grupo de adolescentes y aun preadolescentes más vulnerables a un mal uso de Internet. Sin afectar a la mayoría, ni tener siempre una gravedad extrema, parecen enquistarse las conductas problemáticas en torno a un 25-30\% del alumnado de estas edades y aún menores. Todo ello requiere intensificar los estudios cualitativos que profundicen en el origen y las razones que alimentan estos comportamientos disruptivos en solitario; con el consejo y la guía adecuados de amigos, padres y profesores, se podría promover de ese modo un auténtico uso empoderador de las TIC en las nuevas generaciones digitales del siglo XXI.

Declaración de divulgación del autor: el autor de este manuscrito declara que no existen intereses en conflicto.

\section{Anexos}

Anexo 1. Selección de ítems del cuestionario «Internet, redes sociales y educación».

\begin{tabular}{|c|c|}
\hline $\begin{array}{l}\text { P6. ¿Sueles pensar que es fiable la } \\
\text { información que encuentras en } \\
\text { internet? }\end{array}$ & $\begin{array}{l}\text { Sí } \\
\text { Solo a veces } \\
\text { No }\end{array}$ \\
\hline $\begin{array}{l}\text { P12. ¿Cuento cosas sobre los } \\
\text { demás o emito juicios sobre sus } \\
\text { acciones sin considerar el daño o } \\
\text { disgusto que les puede ocasionar? }\end{array}$ & $\begin{array}{l}\text { Sí } \\
\text { Algunas veces } \\
\text { Casi nunca } \\
\text { Nunca }\end{array}$ \\
\hline $\begin{array}{l}\text { P13. ¿Pueden acceder mis padres a } \\
\text { mi perfil? }\end{array}$ & $\begin{array}{l}\text { Sí } \\
\text { Sí, pero no a mi perfil completo } \\
\text { No }\end{array}$ \\
\hline $\begin{array}{l}\text { P15. ¿Soy consciente de que lo que } \\
\text { publico o cuento (fotos, por } \\
\text { ejemplo) a un grupo de amigos } \\
\text { deja de estar bajo mi control y } \\
\text { puede llegar fácilmente a manos } \\
\text { de otros? }\end{array}$ & $\begin{array}{l}\text { Sí, soy muy consciente } \\
\text { Sí, porque he tenido malas } \\
\text { experiencias con este tema } \\
\text { Sí, la mayoría de las veces } \\
\text { Me da igual porque estoy bastante } \\
\text { seguro de lo que cuelgo } \\
\text { Casi nunca he pensado en ello } \\
\text { No, porque me fío mucho de mis } \\
\text { amigos }\end{array}$ \\
\hline $\begin{array}{l}\text { P20. ¿Qué has hecho cuando te } \\
\text { has sentido amenazado a través de } \\
\text { internet? } \\
\text { (Pueden marcarse varias } \\
\text { respuestas.) }\end{array}$ & $\begin{array}{l}\text { He acudido a alguna de mis } \\
\text { amistades } \\
\text { He hablado con mis padres } \\
\text { He hablado con un adulto de } \\
\text { confianza } \\
\text { Lo he denunciado a la dirección del } \\
\text { centro escolar o a la policía } \\
\text { He preferido no comentarlo con } \\
\text { nadie } \\
\text { Nunca me he sentido amenazado }\end{array}$ \\
\hline $\begin{array}{l}\text { P25. ¿Soy consciente de que } \\
\text { algunas personas de mi edad lo } \\
\text { han pasado realmente mal al } \\
\text { descubrir información, fotos o } \\
\text { vídeos privados en manos de } \\
\text { otros? }\end{array}$ & $\begin{array}{l}\text { Sí, soy muy consciente } \\
\text { Sí, alguna vez lo había pensado } \\
\text { No, rara vez lo pienso } \\
\text { No lo sabía }\end{array}$ \\
\hline $\begin{array}{l}\text { P38. Gracias a la red... (elige las } \\
\text { frases que mejor reflejen lo que } \\
\text { piensas, hasta un máximo de } 2 \text { ) }\end{array}$ & $\begin{array}{l}\text { Me siento más importante } \\
\text { Soy más sociable } \\
\text { Soy más popular } \\
\text { Me cuesta concentrarme cuando } \\
\text { estudio } \\
\text { Estoy de mejor humor } \\
\text { Estoy de peor humor }\end{array}$ \\
\hline $\begin{array}{l}\text { P50. ¿Has comprobado en tu red } \\
\text { social el grado de privacidad que } \\
\text { tienen los datos y las fotos que } \\
\text { pones? }\end{array}$ & $\begin{array}{l}\text { No, nunca me he fijado en eso } \\
\text { Sí, alguna vez lo he mirado pero rara } \\
\text { vez lo he tenido en cuenta } \\
\text { Sí, lo conozco y lo tengo en cuenta }\end{array}$ \\
\hline
\end{tabular}

\section{Referencias}

Alfaro, M., Vázquez, M.E., Fierro, A., Herrero, B., Muñoz, M.F., \& Rodríguez, L. (2015). Uso y riesgos de las tecnologías de la información y comunicación en adolescentes de 13-18 años. Acta Pediátrica Española, 73(6), 126-35.

American Psychiatric Association (2014). Guía de consulta de los criterios diagnósticos del DSM-5®: Spanish Edition of the Desk Reference to the Diagnostic Criteria From DSM-5®. American Psychiatric Pub.

Arnaiz, P., Cerezo, F., Giménez, A.M., \& Maquilón, J. (2016). Conductas de ciberadicción y experiencias de cyberbullying entre adolescentes. Anales de psicología, 32(3), 761-769. http://dx.doi.org/10.6018/ analesps.32.3.217461 
Ávila, T. P., Fernández, C. G., \& Ruiz, E. P. (2019). Hábitos de consumo de las nuevas tecnologías en adolescentes ¿Uso o abuso? European Journal of Child Development, Education and Psychopathology, 7(1), 47-57. http://doi.org/10.30552/ejpad.v7i1.88

Ballesteros, J. C., \& Megías, I. (2015). Jóvenes en la red: un selfie. Centro Reina Sofía sobre Adolescencia y Juventud.

Beland, L-P., \& Murphy, R. (2015). Technology, Distraction \& Student Performance. Center for Economic Performance.

Bernal, A., \& König, K. L. (2017). Percepciones de adolescentes sobre la educación según la identidad personal. Revista Española de Pedagogía, 75(267), 181198. http://doi.org/10.22550/REP752201711

Berríos, L., Buxarrais, M. R., \& Garcés, M. S. (2015). Uso de las TIC y mediación parental percibida por niños de Chile. Comunicar, 22(45), 161-168. http://dx.doi. org/10.3916/C44-2015-17

Besolí, G., Palomas, N., \& Chamarro, A. (2018). Uso del móvil en padres, niños y adolescentes: Creencias acerca de sus riesgos y beneficios. Aloma: Revista de Psicologia, Ciències de l'Educació i de l'Esport, 36(1), 29-39.

Buelga, S., Cava, M. J., \& Musitu, G. (2010). Cyberbullying: victimización entre adolescentes a través del teléfono móvil y de Internet. Psicothema, 22(4), 784789.

Colás, P., De Pablos, J., \& Ballesta, J. (2018). Incidencia de las TIC en la enseñanza en el sistema educativo español: una revisión de la investigación. RED. Revista de Educación a Distancia 56(2). http://dx.doi. org/10.6018/red/56/2

Colás, P., González, T., \& De Pablos, J. (2013) Juventud y redes sociales: Motivaciones y usos preferentes, Comunicar, 40(20), 15-23. http://dx.doi.org/10.3916/ C40-2013-02-01

Chóliz, M. \& Villanueva, V. (2011). Evaluación de la adicción al móvil en la adolescencia. Revista española de drogodependencias, 36(2), 165-183.

Crescenzi, L., Jewitt, C., \& Price, S. (2014). The role of touch in preschool children's learning using iPad versus paper interaction. Australian Journal of Language \& Literacy, 37(2), 86-95.

Díaz-Vicario, A., Mercader, C., \& Gairín, J. (2019). Uso problemático de las TIC en adolescentes. Revista Electrónica de Investigación Educativa, 21, e07, 1-11. http://dx.doi.org/10.24320/redie.2019.21.e07.1882

Espinar, E., \& Fernández, C. (2009). Jóvenes y adolescentes ante las nuevas tecnologías: percepción de riesgos. Athenea Digital, 16, 1-20.

European Union (2015). Being young in Europe today. Eurostat Statistical Books. http://dx.doi.org/10.3916/ C44-2015-12

Fernández-Montalvo, J., Peñalva, A., \& Irazabal, I. (2015). Hábitos de uso y conductas de riesgo en Internet en la preadolescencia. Comunicar, 44(22), 113-20. http://dx.doi.org/10.3916/C44-2015-12

Gairín, J., \& Mercader, C. (2018). Usos y abusos de las TIC en los adolescentes. Revista de Investigación Edu- cativa, 36(1), 125-140. http://dx.doi.org/10.6018/ rie.36.1.284001

García-Martín, S. \& Cantón-Mayo, I. (2019). Uso de tecnologías y rendimiento académico en estudiantes adolescentes. Comunicar 59(27), 73-81. https://doi. org/10.3916/C59-2019-07

Giménez, A. M. (2015). Estrategias de afrontamiento ante el cyberbullying. Una mirada cualitativa desde la perspectiva de los escolares. Campo Abierto, vol. monográfico, 49-65.

Gómez, D. R., Ceacero, D. C., \& Meneses, J. (2018). Usos problemáticos de las TIC entre jóvenes en su vida personal y escolar. Comunicar: Revista científica iberoamericana de comunicación y educación, (56), 91100. https://doi.org/10.3916/C56-2018-09

Grande, M., Cañón, R., \& Cantón, I. (2016). Tecnologías de la información y la comunicación: Evolución del concepto y características. IJERI: International Journal of Educational Research and Innovation, 6, 218-230.

Haddon, L. \& Vincent, J. (Eds.) (2014). European children and their carers' understanding of use, risks and safety issues relating to convergent mobile media. Report D4.1. Unicatt.

$\mathrm{Hu}, \mathrm{C}$. (2017). Students, computers and learning: Where is the connection? Education and Information Technologies, 22(6), 2665-2670. https://doi.org/10.1007/ s10639-017-9670-6

INE. Instituto Nacional de Estadística. (2019). Encuesta sobre equipamiento y uso de tecnologías de información y comunicación en los hogares. Instituto Nacional de Estadística.

Lepicnik, J., \& Samec, P. (2013). Uso de tecnologías en el entorno familiar en niños de cuatro años de Eslovenia. Comunicar, 40, 119-126. http://dx.doi. org/10.3916/C40-2013-03-02

Megías, E. (2019). Una década de cambios en la juventud española. Una lectura (parcial) desde el Centro Reina Sofía sobre Adolescencia y Juventud. METAMORFOSIS, 10(10), 30-51.

Megías, I., \& Rodríguez, E. (2018). Jóvenes en el mundo virtual: usos, prácticas y riesgos. Centro Reina Sofía sobre Adolescencia y Juventud. FAD. Fundación Mapfre.

Melendro, M., García, F.J., \& Goig, R. (2016). El uso de las TIC en el ocio y la formación de los jóvenes vulnerables. Revista Española de Pedagogía, LXXIV, 236, 71-89.

Méndez-Gago, S., \& González-Robledo, L. (2018). Uso y abuso de las Tecnologías de la Información y la Comunicación por adolescentes. Un estudio representativo de la ciudad de Madrid. Universidad Camilo José Cela.

Muñoz-Miralles, R., Ortega-González, R., Batalla-Martínez, C., López-Moron, M. R., Manresa, J. M., \& Torán- Monserrat, P. (2014). Acceso y uso de nuevas tecnologías entre los jóvenes de educación secundaria, implicaciones de salud. Estudio JOITIC. Atención Primaria, 46(2), 77-88. https://doi.org/10.1016/j. aprim.2013.06.001

OECD. Organisation for the Economic Co-operation and Development. (2015). Students, computers and 
learning: Making the connection. Organisation for Economic Co-operation and Development (OECD) Programme for International Student Assessments.

Plaza-de-la-Hoz, J. (2016) Impacto de las redes sociales virtuales en estudiantes adolescentes: Informe de investigación. Revista Internacional de Tecnologías en la Educación, 3(1) 53-63.

Plaza-de-la-Hoz, J. (2018). Ventajas y desventajas del uso adolescente de las TIC: visión de los estudiantes. Revista Complutense de Educación, 29(2), 491-508. http://dx.doi.org/10.5209/RCED.53428

Plowman, L., Stevenson, O., Stephen, C., \& McPake, J. (2012). Preschool children's learning with technology at home. Computers \& Education, 59(1), 30-37. https://doi.org10.1016/j.compedu.2011.11.014

Ramos-Soler, I., López-Sánchez, C., \& Torrecillas-Lacave, T. (2018). Percepción de riesgo online en jóvenes y su efecto en el comportamiento digital. Comunicar, 26(56), 71-79. https://doi.org/10.3916/C56-2018-07

Rial, A., Gómez, P., Braña, T., \& Varela, J. (2014). Actitudes, percepciones y uso de Internet y las redes so- ciales entre los adolescentes de la comunidad gallega (España). Anales de psicología, 30(2), 642-655. https:// doi.org/0.6018/analesps.30.2.159111

Rial, A., Golpe, S., Gómez, P., \& Barreiro, C. (2015). Variables asociadas al uso problemático de Internet entre adolescentes. Health and Addictions, 15(1), 2538.

Rodríguez-Álvarez, J. M., Cabrera-Herrera, M., \& Jiménez, S. Y. (2018). Los riesgos de las TIC en las relaciones entre iguales. Cyberbullying en Educación Primaria y Secundaria. Innoeduca. International Journal of Technology and Educational Innovation, 4(2), 185192. http://dx.doi.org/10.24310/innoeduca.2019. v5i1.35

Valencia-Peris, A., Devís-Devís, J., \& Peiró-Velert, C. (2016). Implicaciones en actividades sedentarias y rendimiento académico en adolescentes: diferencias según variables sociodemográficas. Cultura y Educación: Revista de teoría, investigación y práctica, 28(2), 313-327. 
